\title{
On Halting Process of Quantum Turing Machine
}

\author{
Takayuki Miyadera* and Masanori Ohya** \\ Department of Information Sciences, Tokyo University of Science, Noda City, Chiba 278-8510, Japan
}

(November 1, 2018)

\begin{abstract}
We prove that there is no algorithm to tell whether an arbitrarily constructed Quantum Turing Machine has same time steps for different branches of computation. We, hence, can not avoid the notion of halting to be probabilistic in Quantum Turing Machine. Our result suggests that halting scheme of Quantum Turing Machine and quantum complexity theory based upon the existing halting scheme sholud be reexamined.
\end{abstract}

PACS numbers: 03.67.Lx

In [1] Myers pointed out that there exists a problem in case different branches of quantum computation on a Quantum Turing Machine (QTM) take different numbers of steps to complete their calculation. That is, observation of halting qubit may spoil the computation since it selects a branch of computation and the quantum interference is destroyed after the selection. Subsequently several papers [2-4] on the halting process of QTM were published. In [2] Ozawa proposed a possible solution by means of quantum nondemolition measurement. He showed that if one considers a restricted class of QTMs such that the halting qubit and data slots are not changed after the branch falls into the halting state, the probability to obtain each outcome by a given time does not depend upon the fact whether one employed the halting protocol or not. However there still remains a problem. Even if one employs the protocol proposed, the notion of halting is still probabilistic. That is, a QTM with an input sometimes halts and sometimes does not halt. If one can not get rid of the possibility of such a probabilistic halting, one can not tell anything certain for one experiment since one can not say whether an event of halting or non-halting occured with probability one or just by accident, say with probability $10^{-40}$. Bernstein and Vazirani [5] deals only with QTMs whose branches halt at a same time or none of them halt. If one restrict QTMs to such a class, one does not have the problem anymore. We will show, in the present letter, the restriction is not realistic. That is, we address the following question: for an arbitrarily given QTM and an input, can we tell whether the halting is probabilistic or not? We prove that the answer is negative. It means that there exists no necessary and sufficient condition to prescribe non-probabilistic halting QTMs. Thus our result suggests that the probabilistic halting cannot be avoided and appears naturally.

A QTM [5] consists of a head, a processor and an infinite two-way tape with data slots and other (working) slots. Thus the total Hilbert space is spanned by a complete orthonormalized system $\left\{|x\rangle \otimes|\xi\rangle \otimes\left|q_{j}\right\rangle\right\}$, where $x$ is an infinite sequence of the alphabets $\{B, 0,1\}$ ( $B$ is called as blank) with the condition that the number of nonblank cells is finite and $\xi \in \mathbf{Z}$ represents the head position and $q_{j} \in\left\{q_{0}, q_{1}, \cdots, q_{N}, q_{f}\right\}$ is an internal state. Here $q_{0}$ denotes an initial state and $q_{f}$ a halting state. A QTM is constructed by assigning complex probability amplitudes (components of a unitary matrix) which satisfy local rule condition. That is, only transitions between locally different configurations are allowed. According to [5], the components are assumed to be computable complex number, since otherwise we can not construct the QTM. In QTM, the halting scheme is slightly different from classical Turing machine due to the reduction of wave packet [6]. For each step, we observe whether the internal state is $q_{f}$ or not, in other words, we perform the meaurement of the operator $\left|q_{f}\right\rangle\left\langle q_{f}\right|$. When the outcome is 1, we measure the data slots in the tape and recognize the computation result. When the the outcome is 0 , we do not observe anything and proceed the computation. All the ever known effective computation shemes $[7,8]$ halt with probability one at a certatin time and never halt before then. However for an arbitrarily constructed QTM, the different branches of computation can have different numbers of computation steps in general. In such a case the halting process or the notion of halting itself has the problem as described above. One way to avoid such a difficulty is considering only special type of QTMs and inputs.

We call a pair of a QTM $Q$ and its input $x$ nonprobabilistic halting iff one of the following conditions is satisfied.

i) There exists a $t_{0} \in\{1,2, \cdots\}$ such that at step $t_{0}, Q$ under $x$ halts with probability one and for $s<t_{0}$ it halts with probability zero.

ii) For all the steps, $Q$ under $x$ halts with probability zero.

A pair of $Q$ and $x$ is called probabilistic halting when it is not non-probabilistic. We ask whether there exists an algorithm to judge whether a QTM and an input is probabilistic or not [9].

To answer the above question, we assuume the existence of such an algorithm, (classical) Turing Machine (TM) $T_{0}$. Then we can show contradiction. TM $T_{0}$ works so as to read input $\langle Q, x\rangle$ where $Q$ is a QTM and $x \in\{0,1\}^{*}$ is an input and determine whether $Q$ under the input $x$ is probabilistic halting or not. For reversible TMs, $T_{1}$ and $T_{2}$, let us define a special 
type of QTM $Q\left(T_{1}, T_{2}\right)$ which has two branches running $T_{1}$ and $T_{2}$ without interference as follows. The internal state of $Q\left(T_{1}, T_{2}\right)$ consists of a doubly indexed set $\left\{\left(q_{*}, j\right),\left(q_{0}, j\right),\left(q_{1}, j\right), \cdots,\left(q_{N}, j\right),\left(q_{f}, j\right),\left(q_{* f}, j\right)\right\}$, where $j=1,2$ and $N$ is a sufficiently large number. That is, the Hilbert space of the internal states holds tensor product structure, $\mathbf{C}^{N+4} \otimes \mathbf{C}^{2}$. The internal state is initialized with $\left|q_{*}, 1\right\rangle$ and a halting state is $\left|q_{* f}, 1\right\rangle . Q\left(T_{1}, T_{2}\right)$ with an input $y$ (finite string) behaves as follows:

1) change the internal state from initial state $\left|q_{*}, 1\right\rangle$ to $\frac{1}{\sqrt{2}}\left(\left|q_{0}, 1\right\rangle+\left|q_{0}, 2\right\rangle\right)$

2) for the branch with the second qubit of internal state $|1\rangle$, execute the TM $T_{1}$ under the input $y$ and for the branch of $|2\rangle$, execute $T_{2}$ under the unput $y$.

3) If the internal state is $\left|q_{f}, j\right\rangle(j=1,2)$, change the internal state plus a fixed tape working cell into $\left|q_{* f}, 1\right\rangle \otimes|j\rangle$. (i.e., To satisfy unitarity, the information which branch was lived in is transferred to the tape cell.)

Denote the set of all the QTMs of above type as $S$, i.e., $S:=\left\{Q\left(T_{1}, T_{2}\right) \mid T_{1}, T_{2}\right.$ are reversible TMs $\}$. Since $S$ is a subset of whole set of QTMs, TM $T_{0}$ could determine whether or not $Q\left(T_{1}, T_{2}\right)$ under the input $x$ is probabilistic halting. That is, we can determine that for any given reversible TM $T_{1}$ and $T_{2}$ their computing times for an input $x$ are the same or not. Thus we obtain a TM $T_{0}^{\prime}$ which reads input $\left\langle T_{1}, T_{2}, x\right\rangle$ to compare their computing times, whose output is "Yes" if their computing times are same and otherwise "No".

By use of $T_{0}^{\prime}$, we can construct the following TM $T_{f}$ with its input $\left\langle T_{1}, x\right\rangle$ where $T_{1}$ is a reversible TM and $x$ is its input.

i) Read $T_{1}$ and $x$

ii) Construct a TM $T_{2}$ which never halts under any input iii) input $\left\langle T_{1}, T_{2}, x\right\rangle$ to $T_{0}^{\prime}$

iv) Write the output of iii)

We can see that if the outcome is "Yes" TM $T_{1}$ under the input $x$ does not halt and if the outcome is "No" TM $T_{1}$ under the input $x$ halts. It contradicts the undecidability of halting problem [10] of classical TM. Thus our assertion was proved.

Here we proved that for an arbitrarily constructed QTM we can not say whether it is probabilistic halting or not. The result will suggest that to consider QTMs with diffierent computation steps for each branches is necessary and the notion of halting in QTM should be reexamined again. For instance it may play an important role to construct a quantum version of algorithmic complexity theory.

T.M. thanks Masanao Ozawa, Fumihiko Yamaguchi and Satoshi Iriyama for helpful discussions.
* E-mail: miyadera@is.noda.tus.ac.jp

** E-mail: ohya@is.noda.tus.ac.jp

[1] J. M. Myers, Phys. Rev. Lett. 78 (1997) 1823.

[2] M. Ozawa, Phys. Rev. Lett. 80 (1998) 631. Theoret. Informatics and Appl. 34 (2000) 379.

[3] N. Linden and S. Popescu, quant-ph/9806054

[4] Y. Shi, Phys. Lett. A 293 (2002) 277.

[5] E. Bernstein and U. Vazirani, SIAM Journal on Computing 26, (1997) 1411

[6] D. Deutsch, Proc. Roy. Soc. London Ser. A, 400, (1985) 96

[7] P. W. Shor, SIAM J. Computing, 26 (1997) 1484

[8] L. Grover, Phys. Rev. Lett. 79 (1997) 325

[9] Of course some special QTMs can be shown to be probabilistic or non-probabilistic. However what we want to know is the existence of some universal algorithm independent of QTMs.

[10] R. Penrose, The Emperor's New Mind, Oxford University Press, (1989) 